dividual papers from authors in Australia, Canada, France, Germany, Great Britain, India, Japan and the United States. It is divided into four parts, each of which is introduced by a survey paper, with a most useful selected list of references.

In the first paper in Part 1, entitled "The Lowest Ionosphere", Prof. A. H. Waynick summarizes the recent radio data which may be used as a guide to the possible physical characteristics of the lowest regions of the ionosphere. These characteristics are primarily concerned with the absorption of short waves and their partial reflexion from the lower ionosphere, and the height of reflexion of very long waves. It is pointed out that great advances in our knowledge of the lower ionosphere is likely to occur in the near future. Recent rocket measurements of $\mathrm{X}$-ray intensities in the neighbourhood of $50 \mathrm{~A}$. suggest that this radiation may well be the primary source of energy associated with the formation of the $E$ region. In addition, there now appears to be evidence for postulating the existence of a portion of the $E$ region due to meteoric ionization. The remaining nine papers in Part 1 describe work conducted in this field in Great Britain, North America, Germany and India.

The second part of the report, "Irregularities and Movements in the Ionosphere", opens with a survey of existing knowledge by Mr. J. A. Ratcliffe. At all heights in the ionosphere there are randomly distributed irregularities in the electron density. Information on these irregularities - their size, shape and movement-is obtained from a study of the fading characteristics of waves reflected from, or transmitted through, the appropriate part of the ionosphere. The study of the fading observed at spaced receiving stations has contributed greatly to our knowledge of the movement of these irregularities. During recent years, measurements of the propagation of very-high-frequency waves by scattering from the $D$ and $E$ regions and from auroræe are finding increased application. Observations on the seintillation of radio stars also enable drift movements in the $F^{\prime}$ region to be investigated. The sixteen papers collected with the survey in this part of the report form a very complete and up-to-date appreciation of the state of experimental and theoretical knowledge of this subject.

"A Survey of Present Knowledge of the F'2 Region", by Dr. D. F. Martyn, is the first paper in Part 3 , and describes the salient features of the $F 2$ region, including the production of its ionization, the recombination coefficients, winds and travelling disturbances, and the storms and anomalies associated with this region. While the other eight papers in this section summarize the present state of knowledge, including theoretical speculation, of conditions in the $F 2$ layer, a brief reference is made, in an addendum to the survey, to the wealth of information which was obtained by a rocket ascent in May 1954 to a height of $219 \mathrm{~km}$. A mass-spectrograph was used and data obtained on the composition and pressure in the upper atmosphere. While the results await confirmation, this is undoubtedly an indication of the success of a new technique for exploring the ionosphere.

The fourth and final part of the report deals with "The Mathematics of Wave Propagation through the Ionosphere", and is surveyed by Mr. K. G. Budden. With thirteon other papers, this section describes the mathematical technique used in the study of the properties of the ionosphere, and particularly the variation of ionization density with height, the resulting refractive index and the paths of radio waves in traversing the various ionospheric layers. This section is complementary to the previous three and makes the whole report a comprehensive work of reference which will be of the greatest value to students and research workers for many years to come. The publication of the report must be a very gratifying conclusion to the efforts of $\mathrm{Mr}$. Rateliffe and his colleagues who were responsible for the conception and organization of this conference.

\section{AUXIN AND PROTOPLASMIC STREAMING}

T has now been known for some considerable time that indoleacetic acid and other growth-regulating substances increase the rate of protoplasmic streaming, but no generally accepted explanation of the phenomenon has yet been advanced. J. M. Kelso and J. S. Turner (Austral. J. Biol. Sci., 8, 1, 19; 1955) have now reported on the action of various growth-regulating substances on streaming in single cells of the staminal hairs of Tradescantia virginiana.

As the rate of protoplasmic streaming in these cells is not affected by change of oxygen concentration in a hanging drop enclosing the hairs over the range 1.5-100 per cent of oxygen in the surrounding gas phase, experiments can be carried out without continuous renewal of the water drop. It was observed that, over the physiological range of concentrations, indoleacetic acid, indolebutyric acid, naphthaleneacetic acid and 2,4-dichlorophenoxyacetic acid, all modify the rate of protoplasmic streaming. These substances, added alone, bring about changes in the rate of streaming within 10 min.; maximum effects are reached in $30 \mathrm{~min}$. , and the rates return to the normal in 60-70 min. (exceptions to this rule are noted for naphthaleneacetic acid (high concentrations) and 2,4-dichlorophenoxyacetic acid (low concentrations)). Low concentrations of the auxins stimulate the streaming, high concentrations depress the rate, and intermediate concentrations are without effect.

Auxins thus affect streaming much as they do the growth of stems, the optimal concentrations for stimulation being similar for both processes. The 'total effect' for each growth substance may be estimated as the total extra or diminished distance travelled by a protoplasmic particle in the presence of the applied substance. The maximum positive total effect is given at $1 \mathrm{mgm} . / 1$. (1 p.p.m.) for indoleacetic acid and indolebutyric acid, and at $5 \mathrm{mgm} . / 1$. for naphthaleneacetic acid. There is a marked $p H$ effect for the reaction of streaming to indoleacetic acid, increase in $p \mathrm{H}$ rendering the applied solution much less effective over the whole range of concentration. The temporary effects of indoleacetic acid on streaming, both stimulation and inhibition, are stabilized near their maximal values, if fructose or malic acid is added with the auxin. These results largely confirm those obtained by Thimann and Sweeney for the Avena coleoptile, except that the concentration for indoleacetic acid giving maximal total effect for Avena is as low as $0.01 \mathrm{mgm}$. $/ \mathrm{l}$. Moreover, for Avena, malic acid not only stabilizes the auxin effect but also alters the threshold of response. 